\title{
Detection and Quantification of Resistance of Venturia inaequalis Populations to Sterol Demethylation Inhibitors
}

\author{
Wolfram Köller, W. F. Wilcox, J. Barnard, A. L. Jones, and P. G. Braun
}

First and second authors: Department of Plant Pathology, Cornell University, New York State Agricultural Experiment Station, Geneva 14456; third author: Computer Services, Cornell University, New York State Agricultural Experiment Station, Geneva 14456; fourth author: Department of Botany and Plant Pathology, Michigan State University, East Lansing 48824; fifth author: Agriculture Canada, Research Branch, Kentville, NS, B4N 1J5, Canada.

Accepted for publication 21 October 1996.

\begin{abstract}
Köller, W., Wilcox, W. F., Barnard, J., Jones, A. L., and Braun, P. G. 1997. Detection and quantification of resistance of Venturia inaequalis populations to sterol demethylation inhibitors. Phytopathology 87:184-190.

Monoconidial isolates of Venturia inaequalis were collected in 1990 and 1991 from orchards in New York, Michigan, and Nova Scotia that had never or only sporadically been treated with fungicides acting as sterol demethylation inhibitors (DMIs). Sensitivities of isolates to two representative DMIs (fenarimol and myclobutanil) were determined by a sensitivity test based on the relative growth (RG) of mycelial colonies at one discriminatory dose. Mean isolate sensitivities were not significantly different $(P>0.2)$ for the majority of the populations tested, and all sensitivity data obtained from these sites were combined to provide a baseline distribution of isolate sensitivities for both fenarimol and myclobutanil. The baseline distributions were compared with isolate sensitivities determined for an experimental orchard in Nova Scotia with a documented case of DMI resistance and for a commercial orchard in Michigan

tance. For both DMIs tested and in both treated orchards, frequencies of isolates with RG values $<80$ had decreased or only slightly increased compared to the baseline population. In contrast, frequencies of isolates with $\mathrm{RG}$ values $>80$ had increased more than 20 -fold over baseline levels. Thus, isolates with RG values $>80$ were rated DMI resistant. The validity of a qualitative isolate classification was tested in controlled infection studies. At doses of fenarimol and myclobutanil recommended for commercial control of apple scab, reproduction of a typical sensitive isolate on treated apple leaves was suppressed completely. Lesions caused by a resistant isolate continued to expand and produced abundant conidia. Statistical analysis of orchard sensitivities revealed that the analysis of isolate counts grouped into the categories DMI sensitive or resistant was most indicative in comparisons of orchard sensitivities aimed at detection of practical DMI resistance. A high degree of cross-resistance between fenarimol and myclobutanil indicated that sensitivity tests with one of the DMIs employed as the diagnostic tool in this study can serve as a test for other DMIs.
\end{abstract} with a long history of DMI use and first evidence of practical DMI resis-
Fungicides acting as sterol demethylation inhibitors (DMIs) are widely used to control apple scab, caused by Venturia inaequalis (Cooke) G. Wint. $(10,11,14,20,28)$. Although isolates of $V$. inaequalis with reduced DMI sensitivities were identified as early as 1985 (27), cases of practical resistance, defined as diminished levels of disease control caused by the selection of resistant isolates (9), have not become a widely documented problem under commercial orchard conditions (8). However, unsatisfactory control of apple scab accompanied by increased frequencies of $V$. inaequalis phenotypes with substantially less sensitivity than the baseline mean was reported in a research orchard where DMI fungicides had been tested intensively for over 10 years $(1,2,7)$.

In contrast to other fungicides, such as benzimidazoles with discrete subpopulations of highly resistant phenotypes, the frequency distribution of isolate sensitivities to DMIs in unexposed pathogen populations is continuous in character and ranges from highly sensitive phenotypes to phenotypes considerably less sensitive than the population mean $(9,12,13,23,24,26,30)$. Such continuous sensitivity distributions make it difficult to define the degree of insensitivity of an isolate that will allow its reproduction at DMI doses recommended for commercial disease control. Reproducing phenotypes substantially less sensitive than the average would produce a competitive advantage over fully controlled phenotypes and, thus, would be selected. As soon as frequencies of such in- sufficiently controlled phenotypes reach levels at which total disease control of the entire population becomes unsatisfactory, the criterion of practical resistance is met (9).

According to this accepted definition of practical resistance (9), the sensitivity range indicative of DMI-resistant phenotypes is best defined by comparing frequencies of isolate sensitivities found in unexposed populations with frequencies found at sites with prolonged DMI histories and declining levels of disease control. This goal implies that quantitative monitoring procedures are in place, baseline sensitivity distributions in nontreated orchards have been determined, and sites with poor DMI performances relating to extensive DMI use are available for comparative testing.

For $V$. inaequalis and DMI fungicides, the questions of useful sensitivity tests and baseline sensitivity distributions have been addressed in previous studies $(12,26)$. The relative mycelial growth of 30 to 50 monoconidial isolates tested at a single discriminatory dose of a particular DMI slightly higher than the mean baseline $50 \%$ effective dose $\left(\mathrm{ED}_{50}\right)$ values was proposed as a simplified yet quantitative test of isolate sensitivities $(12,26)$. The current study was conducted to test the merits of the monitoring procedure, establish sensitivities of populations prior to exposure to DMIs, compare these data with sensitivity distributions found at sites with evidence of practical DMI resistance, and evaluate methods of data analysis suitable for comparison of orchard sensitivities.

\section{MATERIALS AND METHODS}

Sensitivity tests. Previously described methods of isolation and propagation of monoconidial isolates of $V$. inaequalis and sensi- 
tivity tests based on both $\mathrm{ED}_{50}$ and relative growth (RG) values were used $(12,26)$. RG, determined with at least two replicates per isolate, was defined as the diameter of a mycelial colony on media amended with discriminatory DMI doses per colony diameter on unamended media $\times 100$. Colony diameters were measured after 5 to 6 weeks of colony growth at $20^{\circ} \mathrm{C}(12,26)$. The discriminatory doses were $0.05 \mu \mathrm{g} \mathrm{ml}^{-1}$ for fenarimol and $0.1 \mu \mathrm{g} \mathrm{ml}^{-1}$ for myclobutanil (12). The number of isolates tested per orchard ranged from 30 to 54, as recommended by Smith et al. (26). Mycelium for sensitivity tests originated from a single conidium isolated from one lesion per sampled leaf, with diseased leaves randomly collected from at least four trees per orchard. These trees had not been treated with DMIs prior to sampling.

Sampling of orchards. Isolates of $V$. inaequalis were collected from a group of isolated apple trees spread over the Montezuma National Wildlife Refuge, Seneca County, NY (26). Because these trees had never been treated with fungicides, the isolates were representative of a wild-type population (26). Diseased leaves were collected during June 1990. Also during June 1990, several commercial orchards located in the Annapolis Valley of Nova Scotia, Canada, that had never been treated with DMIs (2) were sampled.

Eleven commercial orchards in New York and seven in Michigan that had never or only sporadically been treated with DMIs (no more than four treatments in total) were sampled during June 1991. Isolates from 17 orchards were obtained from the first scab lesions emerging on leaves sampled from trees not treated with a DMI. Isolates from one Michigan orchard were obtained from single-ascospore cultures obtained by discharging ascospores over water agar surfaces from overwintered leaf litter collected at the site. Mycelial colonies for sensitivity tests were obtained from randomly selected ascospores.

The orchard populations never or only sporadically treated with DMIs were compared with populations collected from two orchards with indications of practical DMI resistance. Diseased leaves were sampled during June 1991 from nontreated trees in an experimental orchard at the Research Station, Agriculture Canada, Kentville, NS, where DMI resistance was documented previously $(1,2,7)$. Similarly, diseased leaves were sampled from nontreated trees at a commercial orchard in Michigan during June 1995. Fenarimol had been used for scab control in this orchard since 1987. In 1993 and 1994, six treatments per season with fenarimol in mixture with mancozeb had not provided a commercially acceptable level of control.

Greenhouse experiments. An isolate representing the fenarimol sensitivity found most frequently in baseline populations of $V$. inaequalis (JD-33-92; $\mathrm{ED}_{50}=0.04 \mu \mathrm{g} \mathrm{ml}^{-1}$ ) and an isolate substantially less sensitive than the baseline mean (JD5-29-93; $\mathrm{ED}_{50}=0.4$ $\mu \mathrm{g} \mathrm{ml}^{-1}$ ) were used in a greenhouse infection study. Both isolates had been collected from an experimental orchard at the New York State Agricultural Experiment Station, Geneva. The isolates were maintained as mycelium on potato dextrose agar covered with mineral oil and stored at $4{ }^{\circ} \mathrm{C}$. Conidia were prepared from each isolate according to the procedure described by Parker et al. (17).

A preliminary test of the pathogenicity of the two isolates was performed by inoculating 1003 -week-old apple leaves (McIntosh seedlings) with conidial suspensions at $7 \times 10^{5}$ spores per $\mathrm{ml}$. Monoconidial isolates $(n=15)$ obtained from 15 apple scab lesions on the infected leaves of apple seedlings were reevaluated for fenarimol sensitivity. Mean RG values were $42 \pm 8\left(\mathrm{ED}_{50}=\right.$ $\left.0.04 \mu \mathrm{g} \mathrm{ml}^{-1}\right)$ for isolate JD-33-92 and $88 \pm 9\left(\mathrm{ED}_{50}=0.4 \mu \mathrm{g} \mathrm{ml}^{-1}\right)$ for isolate JD5-29-93.

The leaves of McIntosh trees grafted to MM.106 rootstocks growing in clay pots $(30 \mathrm{~cm}$ diameter) were inoculated with conidia derived from diseased seedling leaves infected with either one of the two isolates. Inoculum was prepared by rinsing conidia from sporulating lesions, and conidial densities were adjusted to $7 \times 10^{5}$ conidia per $\mathrm{ml}$ with the aid of a hemacytometer. The leaves were stored at $4{ }^{\circ} \mathrm{C}$ in paper bags prior to preparation of inoculum for infection studies.
Leaves of grafted trees, each with a single actively growing shoot, were uniformly misted with the conidial suspensions with a spray paint gun. Microscope slides were sprayed with the same conidial suspensions, placed in petri dishes, and examined for conidial germination after $24 \mathrm{~h}$. Germination rates were 90 to $100 \%$ throughout the experiments.

Inoculated trees were placed in a mist chamber for $48 \mathrm{~h}$ at $20^{\circ} \mathrm{C}$ and moved to a greenhouse chamber maintained at $\sim 20^{\circ} \mathrm{C}$ with a $16 \mathrm{~h} / 8 \mathrm{~h}$ light/dark cycle. Commercial formulations of fenarimol (Rubigan 1EC, Dow Elanco, Indianapolis, IN) and myclobutanil (Nova 40W; Rohm and Haas, Philadelphia) were applied $96 \mathrm{~h}$ after inoculation by spraying leaves to run-off with a spray paint gun. Concentrations of fungicide solutions were $29 \mu \mathrm{g} \mathrm{ml}^{-1}$ a.i. for fenarimol and $50 \mu \mathrm{g} \mathrm{ml}^{-1}$ a.i. for myclobutanil. Both dosages reflected commercially recommended rates, as described by Kuck et al. (14) and as specified by U.S. label provisions. Treated trees were incubated for $48 \mathrm{~h}$ at $95 \%$ relative humidity before they were returned to the greenhouse chamber.

Disease development was assessed 3 weeks after inoculation. The number of sporulating lesions on the two youngest and fully expanded leaves of each shoot at the time of inoculation were counted. Nonsporulating, chlorotic looking lesions, similar to those described by Schwabe et al. (20), were not counted. Experiments were done three times with independently prepared inoculum and fungicide solutions and with separate sets of three to four potted trees. For data analysis, lesion counts obtained for all three replicates were combined.

Data analysis. Regressions of $\mathrm{RG}$ and $\mathrm{ED}_{50}$ values were derived from previous $\mathrm{ED}_{50}$ calculations (12). For each isolate of $V$. inaequalis analyzed, the $\mathrm{ED}_{50}$ and $\mathrm{RG}$ values reflecting discriminatory doses of $0.05 \mu \mathrm{g} \mathrm{ml} l^{-1}$ for fenarimol and $0.1 \mu \mathrm{g} \mathrm{ml}^{-1}$ for myclobutanil were extrapolated from dose response regressions obtained from sensitivity tests at eight DMI concentrations (12). A total of 11 isolates for fenarimol and 10 isolates for myclobutanil were analyzed.

Means of RG values determined for individual orchard populations were compared according to the nonparametric KolmogorovSmirnov test of SYSTAT, version 5.2 (Systat, Inc., Evanston, IL). The nonparametric test was chosen because some of the orchard sensitivity distributions departed significantly from normality. Categorical fungicide sensitivity data obtained for different populations were compared by fitting log-linear models (16) supported by SYSTAT with numbers of isolates grouped into the categories DMI sensitive and resistant. The model assumes that sensitivity categories are multinomially distributed within orchards, and it evaluates the homogeneity of the distributions, with nonhomogeneity indicating differences in distribution among populations. For the greenhouse infection study, effects of fungicide treatments on the number of sporulating scab lesions were evaluated according to the fully factorial analysis of variance supported by SYSTAT.

\section{RESULTS}

Comparison of RG and $\mathbf{E D}_{\mathbf{5 0}}$ values. The majority of DMI sensitivities of $V$. inaequalis isolates have been determined as $\mathrm{ED}_{50}$ values $(2,4-6,12,18,19,21,26)$. As an alternative, the $\mathrm{RG}$ of mycelial colonies at a single discriminatory DMI dose was proposed as a simplified sensitivity test $(12,26)$. To compare both sensitivity measures, regressions of $\mathrm{ED}_{50}$ and corresponding $\mathrm{RG}$ values were derived for fenarimol and myclobutanil with isolates representative of the entire range of baseline sensitivities. Both measures of DMI sensitivities were highly correlated (Fig. 1). Regression analysis confirmed that $\mathrm{RG}$ values were quantitative measures of isolate sensitivities.

In contrast to $\mathrm{ED}_{50}$ values, $\mathrm{RG}$ values originated from tests conducted at a single discriminatory dose. To determine the margin of error inherent to estimates of $\mathrm{ED}_{50}$ values from measured $\mathrm{RG}$ values, the sensitivity of a single isolate was determined 40 times, with two 
replicates employed in each test. RG values ranged from 31 to 54 , with a mean of 42.5. The distribution of RG values for the single isolate was symmetrical, with $90 \%$ of test values within \pm 6.5 of the mean.

DMI sensitivities in $V$. inaequalis populations prior to DMI exposure. Employing the RG test procedure, sensitivity distributions for fenarimol and myclobutanil were determined for a wildtype population of $V$. inaequalis sampled from the Montezuma Wildlife Refuge and for populations from commercial orchards in Nova Scotia $(n=1)$, New York $(n=11)$, and Michigan $(n=7)$. The commercial sites had never or rarely been treated with DMIs up to the time of sampling.

Based on the nonparametric Kolmogorov-Smirnov test, mean sensitivities to fenarimol determined for 17 of the 19 orchard populations sampled in 1990 in Canada and in 1991 in New York and Michigan were not significantly different $(P>0.2)$ from the Montezuma wild-type population tested in 1990. All isolate sensitivities obtained from the sites with indistinguishable fenarimol sensitivities were combined to provide a typical baseline sensitivity distribution (Table 1). Mean RG values ranged from 37.4 to 42.7.

Results for myclobutanil were similar to those obtained for fenarimol. Mean sensitivities of 15 orchards were not different from the wild-type population $(P>0.2)$, with mean RG values ranging from 36.7 to 43.1 . These isolate sensitivities were combined to a typical baseline distribution (Table 1). The sample sizes employed for determining fenarimol and myclobutanil baseline sensitivities were sufficient to detect rare isolate sensitivities of $0.4 \%$ frequencies with $95 \%$ confidence (15).

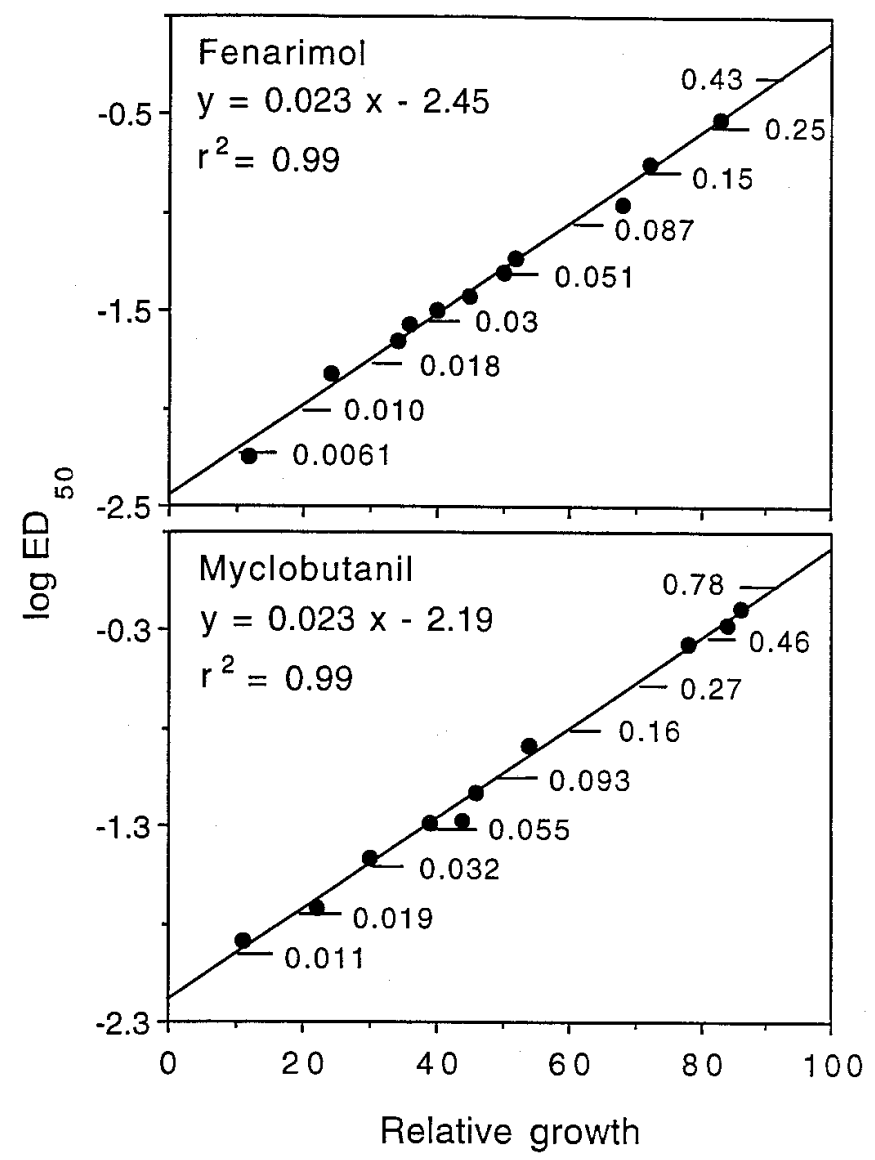

Fig. 1. Regression of $\log 50 \%$ effective dose $\left(\mathrm{ED}_{50}\right)$ values (micrograms per milliliter) obtained from tests at eight sterol demethylation inhibitor fungicide concentrations and relative growth (RG) values corresponding to discriminatory doses of $0.05 \mu \mathrm{g} \mathrm{ml}^{-1}$ (fenarimol) and $0.1 \mu \mathrm{g} \mathrm{ml}^{-1}$ (myclobutanil). $\mathrm{ED}_{50}$ and $\mathrm{RG}$ values corresponding to the discriminatory doses were derived from complete dose-response regressions for 10 to 11 isolates of Venturia inaequalis. Values assigned to horizontal bars are estimated $\mathrm{ED}_{50}$ values (micrograms per milliliter) corresponding to RG values of 10 to 90 .
The baseline sensitivity distributions from nontreated orchards showed a high level of similarity. Mean RG values of baseline sensitivities obtained for fenarimol tested at a discriminatory dose of $0.05 \mu \mathrm{g} \mathrm{ml}^{-1}$ and myclobutanil tested at a discriminatory dose of $0.1 \mu \mathrm{g} \mathrm{ml}^{-1}$ were not different $(P=0.3)$. Moreover, both mean RG values compared well $(P=0.15)$ with baseline data (26) reported previously for the DMI flusilazole, which was tested at a discriminatory dose of $0.01 \mu \mathrm{g} \mathrm{ml}^{-1}$ (Table 1). The isolates for flusilazole sensitivity tests had been sampled in 1987 from the same Montezuma wild-type site and an orchard with a mean flusilazole sensitivity not different $(P=0.27)$ from the wild-type site $(26)$.

Estimated $\mathrm{ED}_{50}$ values (Fig. 1) of typical baseline isolates ranged from $0.004 \mu \mathrm{g} \mathrm{ml}^{-1}$ to $>0.55 \mu \mathrm{g} \mathrm{ml}^{-1}$ for fenarimol and from 0.008 $\mu \mathrm{g} \mathrm{ml} l^{-1}$ to $>0.9 \mu \mathrm{g} \mathrm{ml}^{-1}$ for myclobutanil. Estimated mean $\mathrm{ED}_{50}$ values of baseline populations (Fig. 1) were $0.032 \mu \mathrm{g} \mathrm{ml}^{-1}$ for fenarimol and $0.056 \mu \mathrm{g} \mathrm{m} \mathrm{l}^{-1}$ for myclobutanil. Both values departed only slightly from mean sensitivities determined previously with a small number of representative isolates (12).

Characterization of DMI-resistant phenotypes. According to an operational definition of practical fungicide resistance, isolates of pathogens are resistant if their frequencies have increased substantially at sites with poor fungicide performances (9). An orchard with a documented case of DMI resistance (Nova Scotia) $(1,2,7)$ and another with circumstantial evidence of population shifts toward DMI resistance (Michigan) were selected for comparisons of phenotype sensitivities. For populations of $V$. inaequalis sampled from both orchards and for both DMIs tested, isolates with RG values ranging from 0 to $60 \mathrm{had}$ decreased in frequencies in comparison to the baseline population, whereas isolate frequencies with $\mathrm{RG}$ values $>60$ had increased above baseline levels (Fig. 2). However, the magnitude of frequency increases determined for isolates with $R G$ values $>60$ was not uniform, with the highest increases observed for isolates with RG values $>80$ (Fig. 3). Frequency increases of isolates with intermediate RG values (61 to 80) were small in comparison (Fig. 3).

In compliance with the definition of practical resistance based on substantial frequency increases of resistant phenotypes at sites with unsatisfactory disease control (9), isolates with RG values $>80$ were considered DMI resistant. Considering RG values of 80 as a functional delimitation of sensitive from resistant phenotypes, estimated $\mathrm{ED}_{50}$ values (Fig. 1) reflecting this delimitation were $0.25 \mu \mathrm{g} \mathrm{ml}^{-1}$ for fenarimol and $0.46 \mu \mathrm{g} \mathrm{ml}^{-1}$ for myclobutanil.

Efficacy of apple scab control with DMIs in relation to isolate sensitivities. To test the validity of two categories, DMI sen-

TABLE 1. Typical sensitivity distributions of monoconidial isolates of Venturia inaequalis prior to the exposure of populations to sterol demethylation inhibitor (DMI) fungicides

\begin{tabular}{|c|c|c|c|}
\hline \multirow[b]{2}{*}{$\mathrm{RG}^{\mathrm{a}}$} & \multicolumn{3}{|c|}{ Frequency of isolates $(\%)$} \\
\hline & Fenarimol $^{\mathrm{b}}$ & Myclobutanil $^{\mathrm{b}}$ & Flusilazole $^{\mathrm{b}, \mathrm{c}}$ \\
\hline $0-10$ & 0.8 & 2.1 & 0.5 \\
\hline $11-20$ & 7.8 & 10.5 & 4.6 \\
\hline $21-30$ & 16.6 & 18.8 & 17.0 \\
\hline $31-40$ & 30.6 & 23.1 & 26.8 \\
\hline $41-50$ & 20.6 & 20.3 & 22.1 \\
\hline $51-60$ & 13.6 & 14.7 & 15.0 \\
\hline $61-70$ & 5.8 & 5.4 & 8.8 \\
\hline $71-80$ & 2.5 & 2.9 & 3.6 \\
\hline $81-90$ & 1.2 & 1.4 & 1.0 \\
\hline$>90$ & 0.5 & 0.8 & 0.5 \\
\hline Mean RG & 40.7 & 39.7 & 42.6 \\
\hline$n$ & 748 & 627 & 194 \\
\hline
\end{tabular}

a Relative growth (RG) defined as mean colony diameter on media amended with discriminatory DMI doses per mean colony diameter on unamended media $\times 100$

${ }^{\mathrm{b}}$ Fungicides were tested at discriminatory doses of $0.05 \mu \mathrm{g} \mathrm{ml}^{-1}$ (fenarimol), $0.1 \mu \mathrm{g} \mathrm{ml}^{-1}$ (myclobutanil), and $0.01 \mu \mathrm{g} \mathrm{ml}^{-1}$ (flusilazole).

${ }^{c}$ Data from Smith et al. (26). 
sitive and resistant, derived from frequency increases of isolate sensitivities in DMI-treated orchards, efficacies of fenarimol and myclobutanil were tested in a greenhouse infection study. Apple leaves were inoculated with an isolate sensitive $(\mathrm{RG}=42)$ or resistant $(R G=88)$ to fenarimol. Levels of scab control were determined by treating leaves $96 \mathrm{~h}$ after inoculation as described previously $(20,28)$. Disease severities on nontreated leaves were not different $(P=0.4)$ for the two isolates (Table 2). The sensitive isolate was controlled fully by both fenarimol and myclobutanil (Table 2). Both DMIs significantly $(P<0.001)$ reduced scab severity on leaves inoculated with the resistant isolate, but the number of sporulating lesions was reduced by only $51 \%$ for fenarimol and $75 \%$ for myclobutanil compared to $100 \%$ for the sensitive isolate (Table 2). The slightly higher level of control achieved with myclobutanil compared to fenarimol was of low significance $(P=0.07)$.

Comparison of population sensitivities to DMI fungicides. As described above, mean fenarimol RG values of isolates sampled from 2 of the 19 orchard populations tested were significantly different from the Montezuma wild-type population, regardless of no or minimal exposure to DMI fungicides prior to sampling. Isolates from one of the New York orchards tested were more sensitive than typical (mean $\mathrm{RG}=37 ; P=0.03$ ). Mean $\mathrm{RG}$ values of isolates sampled from a second orchard indicated a significantly less sensitive population (Table 3 ), with frequencies higher than typical in the DMI-sensitive RG range of 61 to 80 (29 versus $8 \%$ ).

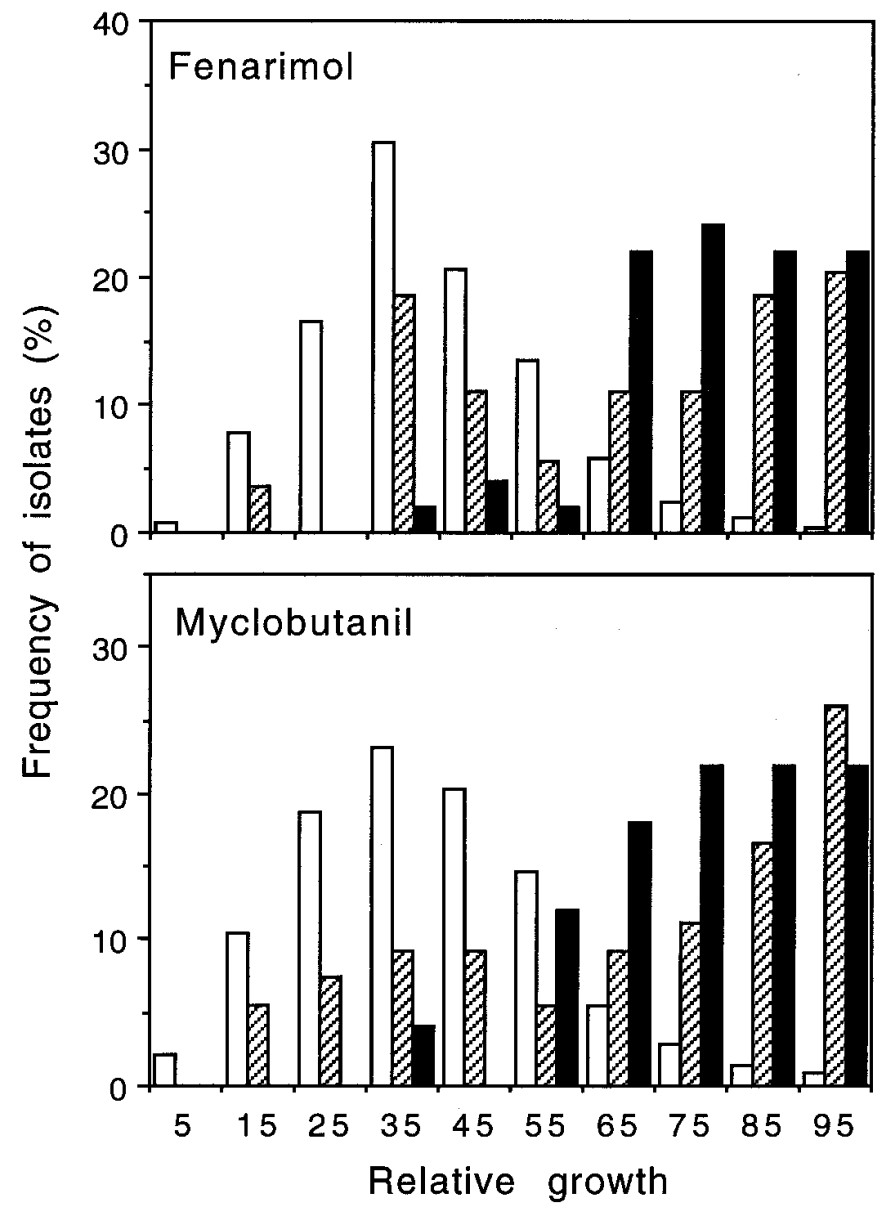

Fig. 2. Frequency distributions of sensitivities of Venturia inaequalis isolates to the sterol demethylation inhibitor (DMI) fungicides fenarimol and myclobutanil. Isolates were sampled from typical nontreated orchards (open bars) or from DMI-treated orchards in Nova Scotia (hatched bars) and Michigan (solid bars), both with first evidence of practical DMI resistance. Relative growth values were determined at discriminatory doses of $0.05 \mu \mathrm{g} \mathrm{ml}^{-1}$ (fenarimol) and $0.1 \mu \mathrm{g} \mathrm{ml}^{-1}$ (myclobutanil).
Similar results were obtained for myclobutanil. Four orchard populations were significantly less sensitive $(P<0.04)$ than isolates from the wild-type site. Surprisingly, sensitivities among the four less sensitive populations were not different from each other $(P>$ $0.3)$. The combined isolate sensitivities exhibited a mean RG value of 46.5 and were significantly different $(P<0.001)$ from the typical baseline sensitivity (Table 3 ). This population, which was not exposed to DMI fungicides but was less sensitive than typical (Table 3), was distinguished by increased frequencies in DMIsensitive RG ranges of 41 to 60 (50 versus 35\%) and 61 to 80 (13

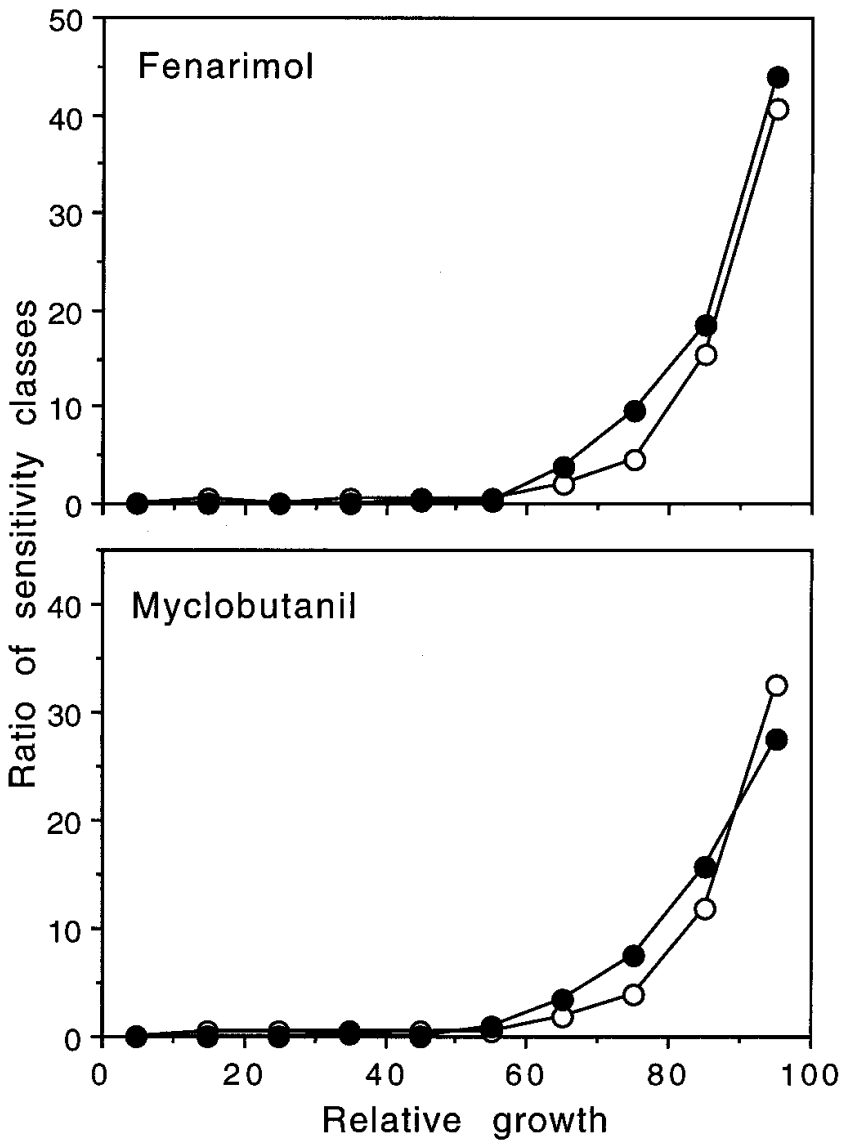

Fig. 3. Comparison of frequencies of Venturia inaequalis isolate sensitivities to the sterol demethylation inhibitor (DMI) fungicides fenarimol and myclobutanil obtained for two DMI-resistant orchards and a typical baseline population. Ratios of sensitivity classes were calculated by dividing isolate frequencies determined for distinct classes of relative growth (RG) values at a resistant orchard in Nova Scotia (open circles) and Michigan (closed circles) by respective frequencies typical for baseline orchards. RG values were determined at discriminatory doses of $0.05 \mu \mathrm{g} \mathrm{ml}^{-1}$ (fenarimol) and $0.1 \mu \mathrm{g} \mathrm{ml}^{-1}$ (myclobutanil).

TABLE 2. Control of apple scab on leaves of McIntosh apple shoots inoculated with a sterol demethylation inhibitor (DMI) fungicide-sensitive or -resistant isolate of Venturia inaequalis and treatment $96 \mathrm{~h}$ after inoculation with the DMI fenarimol or myclobutanil

\begin{tabular}{lccc}
\hline & $\begin{array}{c}\text { Dosage } \\
\text { Fungicide }\end{array}$ & \multicolumn{2}{c}{ Apple scab severity $^{\mathrm{a}}$} \\
\cline { 4 - 4 } Control & $\ldots$ & $59(19)$ & $53(23)$ \\
Fenarimol & 29 & 0 & $26(22)$ \\
Myclobutanil & 50 & 0 & Sensitive isolate $^{\mathrm{b}}$ \\
\hline
\end{tabular}

a Mean number of sporulating lesions per leaf. Values represent means of data combined from three replicates, with six to eight leaves per replicate. Standard deviations are given in parentheses.

${ }^{\mathrm{b}}$ Isolate JD-33-92 sensitive $\left(50 \%\right.$ effective dose $\left[\mathrm{ED}_{50}\right]=0.04 \mu \mathrm{g} \mathrm{ml}^{-1}$; relative growth $[\mathrm{RG}]=42$ ) to fenarimol.

${ }^{c}$ Isolate JD5-29-93 resistant $\left(\mathrm{ED}_{50}=0.4 \mu \mathrm{g} \mathrm{ml}{ }^{-1} ; \mathrm{RG}=88\right)$ to fenarimol. 
versus $8 \%$ ). The sensitivity difference, most likely, was not caused by a random sampling error, because mean RG values of 15 blocks $(n=40)$ of data chosen from the randomly tabulated sensitivities obtained for all typical baseline isolates were not different $(P>0.2)$.

The mean RG values determined for the two DMI-resistant orchards in Nova Scotia and Michigan were significantly different $(P<$ 0.001 ) from both populations never or rarely exposed to DMIs. In this case, the difference was primarily due to substantial frequency increases of DMI-resistant isolates with RG values >80 (Table 3).

Variations of isolate frequencies within the DMI-sensitive RG range of 0 to 80 , as observed for the two populations never exposed to DMIs, were not taken into account in a comparison of isolate counts grouped into the categories DMI sensitive $(\mathrm{RG}=0$ to 80) and resistant ( $R G>80)$. The result of an analysis of respective categorical data showed that sensitivity differences between both populations not exposed to DMIs were not significant, whereas both DMI-resistant sites remained significantly different from both unexposed populations (Table 3). Moreover, differences between the DMI-resistant Nova Scotia and Michigan orchards were not significant $(P>0.3)$, reflecting the initial evidence for practical DMI resistance reported for both sites.

Considering the experimental error of \pm 6.5 inherent to the $R G$ test, isolates with RG values of 73 to 87 could be misclassified as either sensitive or resistant by arbitrarily dividing a continuous distribution of isolate sensitivities into two categories. However, this source of error could be expected to be largely balanced by equal chances of isolate misqualifications. This assumption was confirmed by analyzing the categorical data obtained from all 20 orchards never or sporadically treated with DMI fungicides. With a sample size of 30 to 54 isolates, the number of isolates resistant to fenarimol $(\mathrm{RG}>80)$ ranged from 0 to 2 . Orchard differences of this magnitude were not significant $(P>0.2)$ in a comparison of categorical data. Respective data were identical for myclobutanil.

\section{DISCUSSION}

Detection and quantification of fungicide resistance aid in the diagnosis of resistance as the cause of unsatisfactory disease control, documentation of emerging resistance prior to control failures, and evaluation of relative merits inherent to the options of resistance management strategies (3). For any given pathogen-fungicide system, the prerequisite for this multipurpose task is a sufficiently precise test of isolate sensitivities. Moreover, knowledge of baseline sensitivities prior to the introduction of a fungicide and deter- mination of sample sizes required for the detection of significant population differences are equally important.

Practical use of this information requires a definition of resistance based on the dynamics of population responses to fungicide exposure, confirmation of continued reproduction of isolates classified as resistant on host plants treated with recommended fungicide doses, and determination of the degree of cross-resistance among related members of a particular fungicide class. This list of requirements, which seldom has been explored completely, was thoroughly investigated for $V$. inaequalis and DMI fungicides in this and previous studies $(12,26)$.

The most commonly used tests for determining DMI sensitivities of $V$. inaequalis isolates are based on the inhibition of mycelial growth of monoconidial isolates, with sensitivities expressed as either $\mathrm{ED}_{50}$ values or minimal inhibitory concentrations $(2,4$ $7,12,18,19,21,26,27,29)$. Inhibition of germ tube growth (25) and inhibitory effects of DMIs on developmental stages of the pathogen during conidial infection of apple leaves (22) have been described as alternatives, but thus far, their merits in monitoring DMI resistance have not been documented.

Although isolate $\mathrm{ED}_{50}$ values are the most precise sensitivity measures, their determination requires tests conducted at several DMI concentrations $(12,26)$. To simplify experimental demands, we introduced RG of mycelial colonies at a single DMI dose close to the respective baseline $\mathrm{ED}_{50}$ value as a quantitative measure of isolate sensitivities $(12,26)$. As demonstrated in this study, RG values were correlated with $\mathrm{ED}_{50}$ values, and the $\mathrm{RG}$ test was employed in determining sensitivities of $V$. inaequalis populations to the DMIs fenarimol and myclobutanil.

Similar to the data reported for the DMI flusilazole (26), the baseline sensitivities to fenarimol and myclobutanil that were obtained were distributed continuously, with $\mathrm{ED}_{50}$ values separated by a factor of $>100$. This broad, continuous distribution necessitated an operational definition of DMI resistance based on the response of populations to prolonged DMI exposure. According to an accepted concept of resistance development (9), phenotypes are resistant if their frequencies have increased at sites with unsatisfactory disease control in response to extensive use of a fungicide. We were able to detect such increases by comparing frequencies of isolate sensitivities found in baseline populations with respective frequencies identified at two orchards in Nova Scotia and Michigan, both with first evidence of practical resistance.

At both sites and for both DMIs tested, frequencies of isolates with RG values $>80$ increased substantially over baseline levels,

TABLE 3. Comparison of sterol demethylation inhibitor (DMI) fungicide sensitivities of populations of Venturia inaequalis prior and subsequent to DMI fungicide exposure

\begin{tabular}{|c|c|c|c|c|c|}
\hline \multirow[b]{2}{*}{ Fungicide } & \multirow[b]{2}{*}{ Population parameters } & \multicolumn{4}{|c|}{ Populations } \\
\hline & & Typical baseline $^{a}$ & Less sensitive baseline $^{\mathrm{a}}$ & Resistant, Nova Scotia ${ }^{b}$ & Resistant, Michigan $^{c}$ \\
\hline Fenarimol & 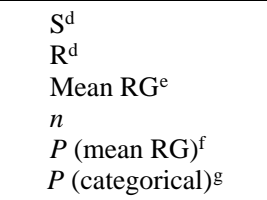 & $\begin{array}{r}98.3 \\
1.7 \\
40.7 \\
748\end{array}$ & $\begin{array}{l}95.4 \\
4.6 \\
53.1 \\
47 \\
<0.001 \\
0.28\end{array}$ & $\begin{array}{l}61.1 \\
38.9 \\
66.4 \\
54 \\
<0.001 \\
<0.001\end{array}$ & $\begin{array}{l}56.0 \\
44.0 \\
78.8 \\
50 \\
<0.001 \\
<0.001\end{array}$ \\
\hline Myclobutanil & 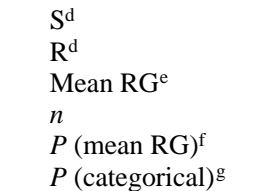 & $\begin{array}{r}97.8 \\
2.2 \\
39.7 \\
627\end{array}$ & $\begin{array}{c}97.2 \\
2.8 \\
46.5 \\
146 \\
<0.001 \\
0.72\end{array}$ & $\begin{array}{l}57.4 \\
42.6 \\
67.2 \\
54 \\
<0.001 \\
<0.001\end{array}$ & $\begin{array}{l}56.0 \\
44.0 \\
78.6 \\
50 \\
<0.001 \\
<0.001\end{array}$ \\
\hline
\end{tabular}

a Isolates were collected from orchards never or rarely treated with DMIs.

b Isolates were collected from an experimental orchard in Nova Scotia with a documented case of DMI resistance (1,2,7).

${ }^{c}$ Isolates were collected from a commercial orchard in Michigan with evidence of DMI resistance.

${ }^{d}$ Frequencies of isolates (percent) in the categories DMI sensitive (S; relative growth $[R G]=0$ to 80$)$ and DMI resistant $(\mathrm{R}$; RG $>80$ ).

e Mean RG of isolates tested at discriminatory doses of $0.05 \mu \mathrm{g} \mathrm{m} l^{-1}$ (fenarimol) and $0.1 \mu \mathrm{g} \mathrm{ml} \mathrm{m}^{-1}$ (myclobutanil).

f Comparison of mean RG values obtained for the typical baseline population with mean RG values of the populations specified (Kolmogorov-Smirnov test).

$\mathrm{g}$ Comparison of typical baseline counts of isolates classified as $\mathrm{S}$ or R with respective counts obtained for the populations specified (log-linear models). 
whereas frequencies with lower RG values had declined or increased only slightly. This difference indicated that isolates with RG values $>80$ had experienced competitive advantages during the prolonged exposure of baseline populations to DMIs. The isolate sensitivity of $\mathrm{RG}=80$, therefore, was defined as the sensitivity distinguishing DMI-sensitive from -resistant phenotypes. This approach of categorizing phenotype sensitivities was validated in a greenhouse infection study with two representative isolates classified as DMI sensitive or resistant. Dosages of fenarimol and myclobutanil recommended for commercial control of apple scab completely controlled the sensitive isolate, whereas the resistant isolate infected the host and produced sporulating lesions. This continued reproduction of the resistant isolate strongly suggests that the frequency shifts detected in the two treated orchards were caused by the selection of such isolates in response to prolonged use of DMI fungicides.

Although the definition of DMI-resistant sensitivities was based on two separate criteria, frequency increases in treated orchards and continued reproduction under controlled infection conditions, the categorical classification of sensitive and resistant phenotypes was, to a certain degree, arbitrary. For example, at both DMIresistant sites, isolates with RG values of 61 to 80 had increased in frequency, although less so than the isolates classified as resistant $(\mathrm{RG}>80)$. However, the relatively high baseline frequencies (8 to $12 \%$ ) of these intermediate phenotypes (Table 1) provided additional evidence that the control of such isolates is satisfactory under commercial conditions. Poor control achieved with such frequent phenotypes would have been noticed in the developmental phase of respective DMIs and probably would have prevented their commercial introduction at the doses eventually recommended for commercial control of apple scab.

The benefit of defining two categories of isolate sensitivities within continuous DMI sensitivity distributions became apparent in the comparative analysis of orchard sensitivities. Comparisons of mean DMI sensitivities revealed that differences could be significant even among populations never or minimally exposed to DMIs. A similar difference not relating to the DMI histories of orchards had been noted previously for the DMI flusilazole (26). However, this type of significant sensitivity variations was confined primarily to the range of isolate sensitivities $(R G=0$ to 80$)$ rated as DMI sensitive. Consequently, these differences did not reflect sensitivity changes of populations with relevance to practical DMI resistance. The reasons for these orchard variations prior to DMI use currently are not understood.

Although a comparison of test data obtained for the two categories, DMI sensitive and resistant, is less comprehensive than the analysis of entire population sensitivities, the approach was more closely adapted to the detection of sensitivity changes with relevance to the level of apple scab control achieved with the DMIs. Differences among the two types of orchards never or rarely exposed to DMIs were insignificant, whereas the differences between all nontreated orchards and the two sites with poor apple scab control in response to prolonged use of DMIs remained highly significant (Table 3).

In a comparison of isolate counts grouped into the categories sensitive and resistant, frequencies of fenarimol- and myclobutanilresistant isolates would become significantly different $(P \leq 0.05)$ from baseline distributions if 5,6, or $8 \%$ of isolates tested were rated resistant with sample sizes of 200, 100, and 50 isolates, respectively. Considering that the frequency of resistant isolates in baseline populations was $2 \%$ and was 41 to $43 \%$ in both orchards with first indications for practical resistance (Table 3), a statistical test with precision in the range of 5 to $8 \%$ appears sufficient for detection of population shifts prior to the emergence of unsatisfactory disease control. Larger sample sizes would be required to precisely detect significant departures from the threshold frequency of $42 \%$ resistant isolates. Frequencies of resistant isolates ranging from 31 to $55 \%, 30$ to $56 \%$, and 26 to $58 \%$ would not be significantly different $(P \geq 0.05)$ from the threshold level with sample sizes of 200,100, and 50 isolates, respectively. However, even this margin of uncertainty appears adequate for orchard evaluations aimed at the magnitude of population shifts toward DMI resistance.

For $V$. inaequalis, a large degree of cross-resistance among the more than 10 members of the DMI family used in apple scab control $(14)$ has been reported before $(7,12,29)$ and was fully confirmed in this study. For example, exclusive treatment of the Michigan orchard with fenarimol had elevated the frequencies of isolates resistant to both fenarimol and myclobutanil to very similar levels (Table 3). Moreover, baseline sensitivity distributions identified in New York, Michigan, and Canada in tests conducted over several years and with three DMIs were not different (Table 1). The high degree of cross-resistance and the conformity of sensitivity distributions determined with different DMIs implies that the data described here and in previous studies $(12,26)$ should be applicable to more general monitoring of DMI sensitivities of $V$. inaequalis populations, with either fenarimol or myclobutanil used as a diagnostic tool.

In this study, we employed a set of procedures for retrieval and analysis of monitoring data with implications beyond the specific case of $V$. inaequalis and DMI fungicides. A quantitative test for isolate sensitivities was developed $(12,26)$, tested, and employed; typical baseline sensitivity distributions were established; isolate sensitivities within a continuous sensitivity distribution were assigned to the categories sensitive and resistant based on population responses to fungicide exposure; the categories were validated in performance studies; and statistical methods of data analysis were adjusted to functional monitoring requirements. These evaluation steps are of particular importance for DMIs but also for existing and future fungicides with broad and continuous distributions of isolate sensitivities within baseline populations.

\section{ACKNOWLEDGMENTS}

This work was supported in part by the U.S. Department of Agriculture (90-34103-539, 94-37313-0678 and TPSU-CU-1484-1071) and the New York State Apple Research and Development Program. We thank J. Burr for technical assistance.

\section{LITERATURE CITED}

1. Braun, P. G. 1994. Development and decline of a population of Venturia inaequalis resistant to sterol-inhibiting fungicides. Norw. J. Agric. Sci. 17 (Suppl.):173-184.

2. Braun, P. G., and McRae, K. B. 1992. Composition of a population of Venturia inaequalis resistant to myclobutanil. Can. J. Plant Pathol. 14: 215-220.

3. Brent, K. 1992. Monitoring fungicide resistance-Purposes, procedures and progress. Pages 1-18 in: Resistance '91: Achievements and Developments in Combating Pesticide Resistance. A. L. Devonshire and D. W. Hollomon, eds. Ellis Science Publishers, London.

4. Carisse, O., and Pelletier, J. R. 1994. Sensitivity distribution of Venturia inaequalis to fenarimol in Québec apple orchards. Phytoprotection 75:35-43.

5. Fiaccadori, R., Gielink, A. J., and Dekker, J. 1987. Sensitivity to inhibitors of sterol biosynthesis in isolates of Venturia inaequalis from Italian and Dutch orchards. Neth. J. Plant Pathol. 93:285-287.

6. Hermann, M., Szith, R., and Zinkernagel, V. 1989. Verringerte Sensitivität einiger Isolate von Venturia inaequalis aus der Steiermark für EBIFungizide. Gartenbauwissenschaft 54:160-165.

7. Hildebrand, P. D., Lockhart, C. L., Newbery, R. J., and Ross, R. G. 1989. Resistance of Venturia inaequalis to bitertanol and other demethylationinhibiting fungicides. Can. J. Plant Pathol. 10:311-316.

8. Hollomon, D. W. 1993. Resistance to azole fungicides in the field. Biochem. Soc. Trans. 21:1047-1051.

9. Köller, W. 1991. Fungicide resistance in plant pathogens. Pages 679-720 in: CRC Handbook of Pest Management in Agriculture, vol. 2. 2nd ed. D. Pimentel, ed. CRC Press, Boca Raton, FL.

10. Köller, W. 1992. Antifungal agents with target sites in sterol functions and biosynthesis. Pages 119-206 in: Target Sites of Fungicide Action. W. Köller, ed. CRC Press, Boca Raton, FL.

11. Köller, W. 1994. Chemical control of apple scab-Status quo and future. 
Norw. J. Agric. Sci. 17 (Suppl.):149-170.

12. Köller, W., Parker, D. M., and Reynolds, K. L. 1991. Baseline sensitivities of Venturia inaequalis to sterol demethylation inhibitors. Plant Dis. 75:726-728.

13. Köller, W., and Scheinpflug, W. 1987. Fungal resistance to sterol biosynthesis inhibitors: A new challenge. Plant Dis. 71:1066-1074.

14. Kuck, K. H., Scheinpflug, H., and Pontzen, R. 1995. DMI fungicides. Pages 205-258 in: Modern Selective Fungicides. 2nd ed. H. Lyr, ed. Gustav Fischer Verlag, Jena, Germany.

15. Leung, H., Nelson, R. J., and Leach, J. E. 1993. Population structure of plant pathogenic fungi and bacteria. Adv. Plant Pathol. 10:157-205.

16. McCullagh, P., and Nelder, J. A. 1989. Generalised Linear Models. Chapman and Hall, London.

17. Parker, D. M., Hilber, U. W., Bodmer, M., Smith, F. D., Yao, C., and Köller, W. 1995. Production and transformation of conidia of Venturia inaequalis. Phytopathology 85:87-91.

18. Parisi, L., Guillaumes, J., and Olivier, J. M. 1990. Variability in the curative effects of sterol demethylation inhibitors towards Venturia inaequalis. Agronomie 10:573-579.

19. Schulz, U. 1991. A method for examining the sensitivity of Venturia inaequalis to DMI fungicides. EPPO Bull. 21:328-329.

20. Schwabe, W. F. S., Jones, A. L., and Jonker, J. P. 1984. Greenhouse evaluation of the curative and protective action of sterol-inhibiting fungicides against apple scab. Phytopathology 74:249-252.

21. Sholberg, P. L., and Haag, P. D. 1993. Sensitivity of Venturia inaequalis isolates from British Columbia to flusilazole and myclobutanil. Can. J. Plant Pathol. 15:102-106.

22. Siebels, C., and Mendgen, K. 1994. A microscopic evaluation of the sensitivity of Venturia inaequalis populations to sterol demethylation inhibi- tors. Mycol. Res. 98:619-624.

23. Skylakakis, G. 1987. Changes in the composition of pathogen populations caused by resistance to fungicides. Pages 227-237 in: Populations of Plant Pathogens: Their Dynamics and Genetics. M. S. Wolfe and C. E. Caten, eds. Blackwell Scientific Publishing, Oxford.

24. Skylakakis, G., and Hollomon, D. W. 1987. Epidemiology of fungicide resistance. Pages 94-103 in: Combating Resistance to Xenobiotics-Biological and Chemical Approaches. M. G. Ford, D. W. Hollomon, B. P. S. Khambay, and R. M. Sawicki, eds. Ellis Horwood, Ltd., Chichester, England.

25. Smith, C. M., Trivellas, A. E., and Johnson, L. E. B. 1991. In vitro methods for monitoring flusilazole sensitivity in populations of Venturia inaequalis (apple scab) and Cercosporidium personatum (peanut late leaf spot). EPPO Bull. 21:323-328.

26. Smith, F. D., Parker, D. M., and Köller, W. 1991. Sensitivity distribution of Venturia inaequalis to flusilazole: Baseline sensitivity and implications for resistance monitoring. Phytopathology 81:392-396.

27. Stanis, V. F., and Jones, A. L. 1985. Reduced sensitivity to sterol inhibiting fungicide in field isolates of Venturia inaequalis. Phytopathology 75: 1098-1101.

28. Szkolnik, M. 1981. Physical modes of action of sterol-inhibiting fungicides against apple diseases. Plant Dis. 65:981-985.

29. Thind, T. S., Clerjau, M., and Olivier, J. M. 1986. First observations on resistance in Venturia inaequalis and Guidnardia bidwellii to ergosterolbiosynthesis inhibitors in France. Proc. Br. Crop Prot. Conf. Pests Dis. 2:491-498.

30. Wolfe, M. S. 1982. Dynamics of the pathogen population in relation to fungicide resistance. Pages 139-148 in: Fungicide Resistance in Crop Protection. J. Dekker and S. G. Georgopoulos, eds. Centre for Agricultural Publishing and Documentation, Wageningen, the Netherlands. 